ANNALES

POLONICI MATHEMATICI

$89.3(2006)$

\title{
On weakly cyclic Ricci symmetric manifolds
}

\author{
by A. A. Shaikh and Sanjib Kumar Jana (Burdwan)
}

\begin{abstract}
We introduce a type of non-flat Riemannian manifolds called weakly cyclic Ricci symmetric manifolds and study their geometric properties. The existence of such manifolds is shown by several non-trivial examples.
\end{abstract}

1. Introduction. The notion of weakly Ricci symmetric manifold was introduced by Tamássy and Binh [4]. A Riemannian manifold $\left(M^{n}, g\right)$ $(n>2)$ is called weakly Ricci symmetric if its Ricci tensor $S$ of type $(0,2)$ is not identically zero and satisfies the condition

$$
\left(\nabla_{X} S\right)(Y, Z)=A(X) S(Y, Z)+B(Y) S(Z, X)+C(Z) S(X, Y),
$$

where $A, B, C$ are 1-forms (not simultaneously zero) and $\nabla$ denotes the operator of covariant differentiation with respect to the metric tensor $g$. Such an $n$-dimensional manifold was denoted by (WRS) $n$. If in (1.1) the 1 -form $A$ is replaced by $2 A$ then the manifold is called a generalized pseudo Ricci symmetric manifold, introduced by Chaki and Koley [1].

A Riemannian manifold $\left(M^{n}, g\right)(n>2)$ is called weakly cyclic Ricci symmetric if its Ricci tensor $S$ of type $(0,2)$ is not identically zero and satisfies

$$
\begin{aligned}
\left(\nabla_{X} S\right)(Y, Z)+ & \left(\nabla_{Y} S\right)(Z, X)+\left(\nabla_{Z} S\right)(X, Y) \\
= & A(X) S(Y, Z)+B(Y) S(Z, X)+C(Z) S(X, Y),
\end{aligned}
$$

where $A, B, C$ are 1-forms (not simultaneously zero). Such an $n$-dimensional manifold will be denoted by (WCRS $)_{n}$.

Section 2 is concerned with some fundamental properties of (WCRS) ${ }_{n}$ and the observation that every (WRS $)_{n}$ is a special type of $(\mathrm{WCRS})_{n}$. But a $(\mathrm{WCRS})_{n}$ is not a $(\mathrm{WRS})_{n}$ in general. However, if the Ricci tensor is of Codazzi type then a (WCRS $)_{n}$ is a (WRS $)_{n}$. It is proved that if a (WCRS $)_{n}$ is a (WRS $)_{n}$ then $-r / 2$ is an eigenvalue of the Ricci tensor $S$ corresponding

2000 Mathematics Subject Classification: 53B50, 53C35.

Key words and phrases: weakly cyclic Ricci symmetric manifold, Einstein manifold, Killing vector field, viscous fluid spacetime. 
to the eigenvector $\mu$ defined by $g(X, \mu)=D(X)$. But in a (WCRS $)_{n}$, if the scalar curvature $r$ is constant then $-r$ is an eigenvalue of the Ricci tensor $S$ corresponding to the eigenvector $\mu$. Also, it is shown that in such a manifold the length of the Ricci tensor $S$ is $r$.

Section 3 is devoted to the Einstein (WCRS) $)_{n}$. It is proved that in an Einstein (WCRS) ${ }_{n}$ the sum of the associated 1-forms is zero everywhere.

Section 4 deals with some global properties of (WCRS $)_{n}$ and it is proved that under certain conditions such a manifold is isometric to a sphere and it does not admit a non-zero Killing vector field, a non-zero projective Killing vector field or a non-zero conformal Killing vector field.

In Section 5 we investigate applications of (WCRS $)_{n}$ to general relativity and cosmology. It is shown that in a viscous fluid (WCRS) ${ }_{4}$ spacetime obeying Einstein's equation with cosmological constant, none of the isotropic pressure and energy density can be a constant and the matter content of such a spacetime is a non-thermalized fluid.

The last section provides several non-trivial examples of (WCRS) ${ }_{n}$ which are not $(\mathrm{WRS})_{n}$.

2. Some fundamental properties of $(\mathrm{WCRS})_{n}(n>2)$. Let $Q$ be the symmetric endomorphism of the tangent space at any point of the manifold corresponding to the Ricci tensor $S$, i.e., $S(X, Y)=g(Q X, Y)$ for all vector fields $X$ and $Y$.

First we consider a $(\mathrm{WRS})_{n}(n>2)$. Interchanging $X, Y, Z$ cyclically in (1.1) and adding the results we obtain

$$
\begin{aligned}
\left(\nabla_{X} S\right)(Y, Z)+ & \left(\nabla_{Y} S\right)(Z, X)+\left(\nabla_{Z} S\right)(X, Y) \\
& =\gamma(X) S(Y, Z)+\gamma(Y) S(Z, X)+\gamma(Z) S(X, Y)
\end{aligned}
$$

where $\gamma(X)=A(X)+B(X)+C(X)$ for all vector fields $X$. Comparing (2.1) with (1.2) we can state the following:

ThEOREM 2.1. A (WRS $)_{n}(n>2)$ is a special type of (WCRS $)_{n}(n>2)$.

If the Ricci tensor is of Codazzi type [3] then

$$
\left(\nabla_{X} S\right)(Y, Z)=\left(\nabla_{Y} S\right)(Z, X)=\left(\nabla_{Z} S\right)(X, Y)
$$

for all $X, Y, Z$, and hence the defining condition of a (WCRS $)_{n}$ reduces to

$$
\left(\nabla_{X} S\right)(Y, Z)=a(X) S(Y, Z)+b(Y) S(Z, X)+c(Z) S(X, Y),
$$

where $a(X)=g\left(X, \frac{1}{3} \varrho_{1}\right), b(X)=g\left(X, \frac{1}{3} \varrho_{2}\right)$ and $c(X)=g\left(X, \frac{1}{3} \varrho_{3}\right)$ are 1 -forms. Thus the manifold is a (WRS $)_{n}$. This leads to the following:

THEOREM 2.2. If the Ricci tensor of a $(\mathrm{WCRS})_{n}(n>2)$ is of Codazzi type then the manifold is a (WRS $)_{n}$. 
Again if a $(\mathrm{WRS})_{n}(n>2)$ is a $(\mathrm{WCRS})_{n}$ then from (1.2) and (2.1) it follows that

$$
\begin{aligned}
{[B(X)+C(X)] S(Y, Z)+[A(Y)+} & C(Y)] S(Z, X) \\
& +[A(Z)+B(Z)] S(X, Y)=0 .
\end{aligned}
$$

Contracting (2.2) with respect to $Y$ and $Z$ we have

$$
r[B(X)+C(X)]+2 A(Q X)+B(Q X)+C(Q X)=0 .
$$

Similarly contracting (2.2) with respect to $Z$ and $X$ we obtain

$$
r[A(Y)+C(Y)]+A(Q Y)+2 B(Q Y)+C(Q Y)=0
$$

for all vector fields $Y$. Replacing $Y$ by $X$ in the last relation we get

$$
r[A(X)+C(X)]+A(Q X)+2 B(Q X)+C(Q X)=0 .
$$

Further, contraction of (2.2) with respect to $X$ and $Y$ yields

$$
r[A(Z)+B(Z)]+A(Q Z)+B(Q Z)+2 C(Q Z)=0
$$

for all vector fields $Z$. Replacing $Z$ by $X$ in the last relation we obtain

$$
r[A(X)+B(X)]+A(Q X)+B(Q X)+2 C(Q X)=0 .
$$

Adding (2.3), (2.4) and (2.5) we get

$$
A(Q X)+B(Q X)+C(Q X)=-\frac{r}{2}[A(X)+B(X)+C(X)],
$$

which can be written as

$$
D(Q X)=-\frac{r}{2} D(X)
$$

where $D(X)=A(X)+B(X)+C(X)$. Let $\mu$ be the vector field associated with the 1-form $D$, i.e., $g(X, \mu)=D(X)$. Hence from (2.6) we have

$$
S(X, \mu)=-\frac{r}{2} g(X, \mu) .
$$

Thus we can state the following:

TheOREM 2.3. If we have (1.2) and (2.1), then $-r / 2$ is an eigenvalue of the Ricci tensor $S$ corresponding to the eigenvector $\mu$.

Let $\left\{e_{i}: i=1, \ldots, n\right\}$ be an orthonormal basis of the tangent space at any point of the manifold. Then setting $Y=Z=e_{i}$ in (1.2) and summing over $i, 1 \leq i \leq n$, we obtain

$$
2 d r(X)=r A(X)+B(Q X)+C(Q X)
$$

where $r$ is the scalar curvature of the manifold. Similarly, contracting (1.2) with respect to $Z$ and $X$ we have

$$
2 d r(Y)=A(Q Y)+r B(Y)+C(Q Y)
$$

for all vector fields $Y$. Replacing $Y$ by $X$ in the above relation we get

$$
2 d r(X)=A(Q X)+r B(X)+C(Q X) .
$$


Further, contraction of (1.2) with respect to $X$ and $Y$ yields

$$
2 d r(Z)=A(Q Z)+B(Q Z)+r C(Z)
$$

for all vector fields $Z$. Replacing $Z$ by $X$ in the above relation we obtain

$$
2 d r(X)=A(Q X)+B(Q X)+r C(X) .
$$

From (2.7) and (2.8), it follows that

$$
H_{1}(Q X)=-r H_{1}(X)
$$

where $H_{1}(X)=A(X)-B(X)$. Let $\mu_{1}$ be the vector field associated with the 1-form $H_{1}(X)$ defined by $g\left(X, \mu_{1}\right)=H_{1}(X)$. Thus (2.10) implies that

$$
S\left(X, \mu_{1}\right)=-r g\left(X, \mu_{1}\right) \text {. }
$$

This leads to the following:

THEOREM 2.4. In a (WCRS $)_{n},-r$ is an eigenvalue of the Ricci tensor $S$ corresponding to the eigenvector $\mu_{1}$.

Similarly, from (2.8) and (2.9) we have

$$
H_{2}(Q X)=-r H_{2}(X)
$$

where $H_{2}(X)=B(X)-C(X)$. Let $\mu_{2}$ be the vector field associated with the 1-form $H_{2}(X)$ defined by $g\left(X, \mu_{2}\right)=H_{2}(X)$. Thus (2.11) implies that

$$
S\left(X, \mu_{2}\right)=-r g\left(X, \mu_{2}\right) \text {. }
$$

Hence we have the following:

THEOREM 2.5. In a (WCRS $)_{n},-r$ is an eigenvalue of the Ricci tensor $S$ corresponding to the eigenvector $\mu_{2}$.

Now addition of (2.7), (2.8) and (2.9) yields

$$
6 d r(X)=r D(X)+D(Q X)
$$

where $D(X)=A(X)+B(X)+C(X)$ for all vector fields $X$. This leads to the following:

Proposition 2.1. In a $(\mathrm{WCRS})_{n}(n>2)$ the relation (2.12) holds.

Now if we assume the scalar curvature to be constant, then (2.12) reduces to

$$
D(Q X)=-r D(X), \quad \text { i.e., } \quad S(X, \mu)=-r g(X, \mu) .
$$

Thus we can state the following:

THEOREM 2.6. In a (WCRS $)_{n}$ with constant scalar curvature, $-r$ is an eigenvalue of the Ricci tensor $S$ corresponding to the eigenvector $\mu$.

We now prove the following lemmas. 
Lemma 2.1. In a (WCRS $)_{n}$, the Ricci tensor $S$ is of the following form:

$$
S=r \alpha \otimes \alpha
$$

where $\alpha$ is a non-zero 1-form defined by $\alpha(X)=g(X, \varrho)$, $\varrho$ being a unit vector field.

Proof. Interchanging $Y$ and $Z$ in (1.2) we obtain

$$
\begin{aligned}
&\left(\nabla_{X} S\right)(Z, Y)+\left(\nabla_{Z} S\right)(Y, X)+\left(\nabla_{Y} S\right)(X, Z) \\
&=A(X) S(Z, Y)+B(Z) S(Y, X)+C(Y) S(X, Z) .
\end{aligned}
$$

Subtracting this relation from $(1.2)$ we get

$$
[B(Y)-C(Y)] S(X, Z)=[B(Z)-C(Z)] S(X, Y)
$$

where the symmetry property of $S$ has been used. Let us consider $E(X)=$ $g(X, \lambda)=B(X)-C(X)$ for all vector fields $X$ where $\lambda$ is a vector field associated with the 1 -form $E$. Then the above relation reduces to

$$
E(Y) S(Z, X)=E(Z) S(X, Y) .
$$

Contraction of (2.14) with respect to $X$ and $Z$ yields

$$
r E(Y)=E(Q Y) \text {. }
$$

Also from (2.14) we have

$$
E(\lambda) S(X, Y)=E(Y) S(X, \lambda)=E(Y) g(Q X, \lambda)=E(Y) E(Q X),
$$

which, in view of (2.15), yields

$$
S(X, Y)=\frac{r}{E(\lambda)} E(X) E(Y)=r \alpha(X) \alpha(Y),
$$

where $\alpha(X)=g(X, \varrho)=(1 / \sqrt{E(\lambda)}) E(X), \varrho$ being a unit vector field associated with the 1 -form $\alpha$. Hence the lemma is proved.

LEMma 2.2. In a (WCRS $)_{n}$, the length of the Ricci tensor $S$ is $r$.

Proof. Let $s^{2}$ be the square of the length of the Ricci tensor $S$. Then from (2.16) we obtain

$$
\begin{aligned}
s^{2} & =\sum_{i=1}^{n} S\left(Q e_{i}, e_{i}\right)=r \sum_{i=1}^{n} \alpha\left(Q e_{i}\right) \alpha\left(e_{i}\right) \\
& =r \sum_{i=1}^{n} g\left(Q e_{i}, \varrho\right) g\left(e_{i}, \varrho\right)=r g(Q \varrho, \varrho)=r^{2},
\end{aligned}
$$

i.e., $s=r$. This proves the lemma.

3. Einstein $(\mathrm{WCRS})_{n}(n>2)$. This section deals with a $(\mathrm{WCRS})_{n}$ defined by (1.2) which is an Einstein manifold. Then we have

$$
S(X, Y)=\frac{r}{n} g(X, Y),
$$


from which it follows that

$$
d r(X)=0 \quad \text { and } \quad\left(\nabla_{Z} S\right)(X, Y)=0
$$

for all vector fields $X, Y, Z$. In view of (3.1) and (3.2), from (1.2) we get

$$
r[A(X) g(Y, Z)+B(Y) g(Z, X)+C(Z) g(X, Y)]=0,
$$

which implies, on contraction with respect to $Y$ and $Z$, that

$$
r[n A(X)+B(X)+C(X)]=0 .
$$

Again contracting (3.3) with respect to $Z$ and $X$, we obtain

$$
r[A(Y)+n B(Y)+C(Y)]=0
$$

for all vector fields $Y$. Replacing $Y$ by $X$ in the last relation we obtain

$$
r[A(X)+n B(X)+C(X)]=0 .
$$

Further, contracting (3.3) with respect to $X$ and $Y$, we obtain

$$
r[A(Z)+B(Z)+n C(Z)]=0
$$

for all vector fields $Z$. Replacing $Z$ by $X$ in the last relation we obtain

$$
r[A(X)+B(X)+n C(X)]=0 .
$$

Adding (3.4), (3.5) and (3.6) we obtain

$$
r(n+2)[A(X)+B(X)+C(X)]=0,
$$

which implies

$$
A(X)+B(X)+C(X)=0,
$$

since $r \neq 0$. If $r=0$ then (3.1) yields $S(X, Y)=0$, which is inadmissible by the definition of (WCRS $)_{n}$. Thus we have the following:

THEOREM 3.1. In an Einstein (WCRS $)_{n}$ the sum of the associated 1forms vanishes everywhere.

Now from (1.2) we have

$$
\left(\nabla_{X} S\right)(X, X)=\frac{1}{3}[A(X)+B(X)+C(X)] S(X, X) .
$$

Therefore, by (3.7), equation (3.8) reduces to

$$
\left(\nabla_{X} S\right)(X, X)=0
$$

which implies that the Ricci curvature $S(X, X)$ is covariantly constant in the direction of $X$. Further, if (3.9) holds then (3.8) implies

$$
A(X)+B(X)+C(X)=0 \quad \text { provided that } S(X, X) \neq 0 .
$$

This leads to the following:

THEOREM 3.2. In an Einstein (WCRS) $n$ with non-vanishing Ricci curvature, the sum of the associated 1-forms is zero if and only if the Ricci curvature $S(X, X)$ is covariantly constant in the direction of $X$. 
4. Some global properties of (WCRS $)_{n}(n>2)$. This section is concerned with compact, orientable $(\mathrm{WCRS})_{n}(n>2)$ without boundary. We prove the following:

TheOREM 4.1. A compact, orientable $(\mathrm{WCRS})_{n}(n>2)$ without boundary of constant scalar curvature is isometric to a sphere provided that it admits a non-isometric conformal transformation.

Proof. We assume that the scalar curvature of a compact, orientable $(\mathrm{WCRS})_{n}(n>2)$ without boundary is constant. Then, in view of $(2.17)$ the length $s$ of the Ricci tensor $S$ is constant. We also suppose that the manifold under consideration admits a non-isometric conformal transformation generated by a vector field $X$. Hence we have

$$
\mathcal{L}_{X} s^{2}=0
$$

where $\mathcal{L}$ is the operator of Lie differentiation. By [6], $M$ is isometric to a sphere. This proves the theorem.

THEOREM 4.2. In a compact, orientable (WCRS $)_{n}(n>2)$ without boundary there exists no non-zero Killing vector field.

Proof. It is known [5] that for a vector field $X$ in a Riemannian manifold $M$, the following relation holds:

$$
\int_{M}\left[S(X, X)-|\nabla X|^{2}-(\operatorname{div} X)^{2}\right] d v \leq 0
$$

where $d v$ denotes the volume element of $M$. If $X$ is a Killing vector field, then $\operatorname{div} X=0$ (see [6]). Hence (4.1) takes the form

$$
\int_{M}\left[S(X, X)-|\nabla X|^{2}\right] d v=0 .
$$

Let $\theta$ be the angle between the vector field $\varrho$ associated with the 1-form $\alpha$ and any vector $X$ of $(\text { WCRS })_{n}$. Then $\cos \theta=g(X, \varrho) / \sqrt{g(X, X)} \leq 1$. Therefore $g(X, \varrho) \leq \sqrt{g(X, X)}$ and consequently from (1.2) it follows that

$$
S(X, X) \leq|r||X|^{2} .
$$

Hence

$$
\int_{M=(\mathrm{WCRS})_{n}}\left[|r||X|^{2}-|\nabla X|^{2}\right] d v \geq \int_{M}\left[S(X, X)-|\nabla X|^{2}\right] d v,
$$

which implies by (4.2) that

$$
\int_{M}\left[|r||X|^{2}-|\nabla X|^{2}\right] d v \geq 0
$$

which in turn, by virtue of (4.1) and (4.2), leads to

$$
\int_{M}\left[|r||X|^{2}-|\nabla X|^{2}\right] d v=0 .
$$

Hence $X=0$. This proves the theorem. 
Definition $4.1([6])$. A vector field $X$ is said to be a projective Killing vector field in a Riemannian manifold $\left(M^{n}, g\right)(n>2)$ if it satisfies

$$
\left(\mathcal{L}_{X} \nabla\right)(Y, Z)=\omega(Y) Z+\omega(Z) Y
$$

for any vector fields $Y$ and $Z$, where $\omega$ is a projective Killing form and $\mathcal{L}$ is the operator of Lie differentiation.

THEOREM 4.3. In a compact, orientable (WCRS $)_{n}(n>2)$ without boundary, there exists no non-zero projective Killing vector field provided that the Ricci curvature is negative.

Proof. We know [5] that for a vector field $X$ in a Riemannian manifold $M$, the following relation holds:

$$
\int_{M}\left[S(X, X)-\frac{1}{4}|d \xi|^{2}-\frac{n-1}{2(n+1)}(\operatorname{div} X)^{2}\right] d v=0,
$$

where $\xi$ is a 1 -form corresponding to the vector field $X$. We now assume that $S(X, X)<0$ and hence from (4.4) we obtain $d \xi=0$ and $\operatorname{div} X=0$. This implies that $X$ is harmonic as well as a Killing vector field. This proves the theorem.

Definition $4.2([6])$. A vector field $X$ is said to be a conformal Killing vector field in a Riemannian manifold $\left(M^{n}, g\right)(n>2)$ if it satisfies

$$
\mathcal{L}_{X} g=2 \psi g
$$

for any vector field $X$, where $\psi$ is given by $\psi=n^{-1}(\operatorname{div} X)$ and $\mathcal{L}$ is the operator of Lie differentiation.

THEOREM 4.4. In a compact, orientable $(\mathrm{WCRS})_{n}(n>2)$ without boundary there exists no non-zero conformal Killing vector field provided that the Ricci curvature is negative.

Proof. It is known from [5] that for a vector field $X$ in a Riemannian manifold $M$, the following relation holds:

$$
\int_{M}\left[S(X, X)-|\nabla X|^{2}-\frac{n-2}{n}(\operatorname{div} X)^{2}\right] d v=0 .
$$

Now we assume that $S(X, X)<0$. Then proceeding as before we obtain

$$
\nabla X=0, \quad \operatorname{div} X=0 .
$$

This proves the theorem.

TheOREM 4.5. In a compact, orientable (WCRS $)_{n}(n>2)$ without boundary, any harmonic vector field is orthogonal to the vector field $\varrho$ associated with the 1-form $\alpha$ and also it is a parallel vector field provided that the Ricci curvature and the scalar curvature are positive. 
Proof. It is known from [5] that for a vector field $X$ in a Riemannian manifold $M$, the following relation holds:

$$
\int_{M}\left[S(X, X)+|\nabla X|^{2}\right] d v=0 .
$$

Now we assume that $S(X, X)>0$ and hence (4.6) reduces to

which implies that

$$
\int_{M}\left[r|g(X, \varrho)|^{2}+|\nabla X|^{2}\right] d v=0
$$

$$
g(X, \varrho)=0, \quad \nabla X=0 .
$$

Hence the theorem follows.

5. General relativistic viscous fluid (WCRS) ${ }_{4}$ spacetime. A viscous fluid spacetime is a connected semi-Riemannian manifold $\left(M^{4}, g\right)$ with signature $(-,+,+,+)$. In general relativity, the key role is played by Einstein's equation

$$
S(X, Y)-\frac{r}{2} g(X, Y)+\lambda g(X, Y)=k T(X, Y)
$$

for all vector fields $X, Y$, where $S$ is the Ricci tensor of type $(0,2), r$ is the scalar curvature, $\lambda$ is the cosmological constant, $k$ is the gravitational constant and $T$ is the energy-momentum tensor of type $(0,2)$. The matter content of the spacetime is described by the energy-momentum tensor $T$ which is to be determined from physical considerations dealing with the distribution of matter and energy.

Let us consider the energy-momentum tensor $T$ of a viscous fluid spacetime in the following form (cf. [2]):

$$
T(X, Y)=p g(X, Y)+(\sigma+p) \alpha(X) \alpha(Y)
$$

where $\sigma, p$ are the energy density and isotropic pressure respectively, and $\alpha$ is the 1-form associated with the unit timelike flow vector field $\varrho$ of the fluid given by $g(X, \varrho)=\alpha(X)$ for all $X$. Then because of (5.2), equation (5.1) can be written as

$$
S(X, Y)=(r / 2+k p-\lambda) g(X, Y)+k(\sigma+p) \alpha(X) \alpha(Y) .
$$

Setting $Y=\varrho$ in (5.3) we deduce by (2.13) that

$$
\sigma=\frac{3 r-2 \lambda}{2 k}
$$

Again contracting (5.3) we get

$$
p=\frac{r+6 \lambda}{6 k} .
$$

We now assume that the scalar curvature $r$ of the spacetime is constant. Then from (5.4) and (5.5), it follows that $\sigma$ and $p$ are constants. Hence we can state the following: 
THEOREM 5.1. If a viscous fluid (WCRS) ${ }_{4}$ spacetime obeys Einstein's equation with cosmological constant then the energy density and isotropic pressure of the fluid are constants provided that the scalar curvature of the spacetime is constant.

Now since $\sigma>0$ and $p>0$, we see from (5.4) and (5.5) that

and hence

$$
\lambda<3 r / 2 \text { and } \lambda>-r / 6
$$

$$
-r / 6<\lambda<3 r / 2 \text {. }
$$

This leads to the following:

THEOREM 5.2. In a viscous fluid (WCRS) ${ }_{4}$ spacetime obeying Einstein's equation, the cosmological constant $\lambda$ satisfies the relation (5.6).

We now discuss whether a viscous fluid (WCRS) $)_{4}$ spacetime with $\varrho$ as the unit timelike flow vector field can admit heat flux or not. Therefore, if possible, let the energy-momentum tensor $T$ be of the following form (cf. [2]):

$$
T(X, Y)=p g(X, Y)+(\sigma+p) \alpha(X) \alpha(Y)+\alpha(X) \beta(Y)+\alpha(Y) \beta(X),
$$

where $\beta(X)=g(X, V)$ for all vector fields $X$, and $V$ is the heat flux vector field, and $\sigma, p$ are the energy density and isotropic pressure respectively. Thus we have $g(\varrho, V)=0$, i.e., $\beta(\varrho)=0$. Hence by the last relation, (5.1) yields

$$
\begin{aligned}
& S(X, Y)-\frac{r}{2} g(X, Y)+\lambda g(X, Y) \\
& \quad=k[p g(X, Y)+(\sigma+p) \alpha(X) \alpha(Y)+\alpha(X) \beta(Y)+\alpha(Y) \beta(X)] .
\end{aligned}
$$

Setting $Y=\varrho$ in (5.7) we deduce by (2.13) that

$$
(-3 r / 2+k \sigma+\lambda) \alpha(X)=-k \beta(X) \quad \text { for all } X \text {. }
$$

Putting $X=U$ in (5.8) we obtain

$$
-3 r / 2+k \sigma+\lambda=0 .
$$

Using the last relation in (5.8) we obtain

$$
\beta(X)=0, \quad \text { since } k \neq 0 .
$$

Thus we have the following:

ThEOREM 5.3. A viscous fluid (WCRS) 4 spacetime obeying Einstein's equation cannot admit heat flux.

6. Some examples of (WCRS $)_{n}(n>2)$. This section deals with several non-trivial examples of (WCRS) ${ }_{n}$. On the real number space $\mathbb{R}^{n}$ (with coordinates $\left.x^{1}, \ldots, x^{n}\right)$ we define a suitable Riemannian metric $g$ such that $\mathbb{R}^{n}$ becomes a Riemannian manifold $\left(M^{n}, g\right)$. We calculate the components of the Ricci tensor and then we verify the defining condition (1.2). 
ExAmple 1. We define a Riemannian metric $g$ on $\mathbb{R}^{4}$ by the formula (6.1)

$$
\begin{aligned}
d s^{2} & =g_{i j} d x^{i} d x^{j} \\
& =e^{x^{1}}\left(d x^{1}\right)^{2}+\left(x^{1}\right)^{2}\left(d x^{2}\right)^{2}+\left(x^{1} \sin x^{2}\right)^{2}\left(d x^{3}\right)^{2}+p^{2}\left(d x^{4}\right)^{2}
\end{aligned}
$$

$(i, j=1,2,3,4)$, where $p$ is an arbitrary constant and $0<x^{1}<1$. Then the only non-vanishing components of the Christoffel symbols and the Ricci tensor and its covariant derivatives are as follows:

$$
\begin{aligned}
\Gamma_{11}^{1} & =\frac{1}{2}, \quad \Gamma_{12}^{2}=\Gamma_{13}^{3}=\frac{1}{x^{1}}, \quad \Gamma_{22}^{1}=-x^{1} e^{-x^{1}}, \quad \Gamma_{33}^{2}=-\sin x^{2} \cos x^{2}, \\
\Gamma_{23}^{3} & =\cot x^{2}, \quad \Gamma_{33}^{1}=-x^{1} e^{-x^{1}}\left(\sin x^{2}\right)^{2}, \\
S_{11} & =-\frac{1}{x^{1}}, \quad S_{22}=e^{-x^{1}}\left(1-\frac{x^{1}}{2}\right)-1, \\
S_{33} & =\left(\sin x^{2}\right)^{2}\left[e^{-x^{1}}\left(1-\frac{x^{1}}{2}\right)-1\right], \\
S_{11,1} & =\frac{1}{\left(x^{1}\right)^{2}}+\frac{1}{x^{1}}, \quad S_{22,1}=e^{-x^{1}}\left(\frac{x^{1}}{2}-\frac{1}{2}-\frac{2}{x^{1}}\right)+\frac{2}{x^{1}}, \\
S_{33,1} & =\left(\sin x^{2}\right)^{2}\left[e^{-x^{1}}\left(\frac{x^{1}}{2}-\frac{1}{2}-\frac{2}{x^{1}}\right)+\frac{2}{x^{1}}\right]
\end{aligned}
$$

where "," denotes the covariant differentiation with respect to the metric tensor $g$. It can be easily found that the scalar curvature of the resulting manifold $\left(M^{4}, g\right)$ is non-zero. We shall now show that $M^{4}$ is a (WCRS) ${ }_{4}$, i.e., it satisfies the defining condition (1.2).

The associated 1-forms are as follows:

$$
\begin{aligned}
& A_{i}(x)= \begin{cases}\frac{x^{1}\left(x^{1}-1\right)+4\left(e^{x^{1}}-1\right)}{x^{1}\left(2-x^{1}-e^{x^{1}}\right)} & \text { for } i=1, \\
0 & \text { otherwise }\end{cases} \\
& B_{i}(x)= \begin{cases}\frac{x^{1}\left(x^{1}-1\right)+4\left(e^{x^{1}}-1\right)}{x^{1}\left(e^{x^{1}}+x^{1}-2\right)} & \text { for } i=1, \\
0 & \text { otherwise }\end{cases} \\
& C_{i}(x)= \begin{cases}-\frac{3}{x^{1}}\left(x^{1}+1\right) & \text { for } i=1, \\
0 & \text { otherwise }\end{cases}
\end{aligned}
$$

at any point $x \in M$. In our $M^{4},(1.2)$ reduces to the following equations:

$$
\begin{aligned}
& S_{11,1}+S_{11,1}+S_{11,1}=A_{1} S_{11}+B_{1} S_{11}+C_{1} S_{11}, \\
& S_{22,1}+S_{21,2}+S_{12,2}=A_{1} S_{22}+B_{2} S_{21}+C_{2} S_{12}, \\
& S_{33,1}+S_{31,3}+S_{13,3}=A_{1} S_{33}+B_{3} S_{31}+C_{3} S_{13},
\end{aligned}
$$

since for the other cases the components of each term of (1.2) vanish identically and (1.2) holds trivially. Now from (6.2)-(6.4) we get the following 
relations for the right hand sides (R.H.S.) and left hand sides (L.H.S.):

$$
\begin{aligned}
\text { R.H.S. of (i) } & =A_{1} S_{11}+B_{1} S_{11}+C_{1} S_{11}=\frac{1}{\left(x^{1}\right)^{2}}+\frac{1}{x^{1}} \\
& =S_{11,1}+S_{11,1}+S_{11,1}=\text { L.H.S. of }(\mathrm{i}) \\
\text { R.H.S. of (ii) } & =A_{1} S_{22}+B_{2} S_{21}+C_{2} S_{12}=e^{-x^{1}}\left(\frac{x^{1}}{2}-\frac{1}{2}-\frac{2}{x^{1}}\right)+\frac{2}{x^{1}} \\
& =S_{22,1}+S_{21,2}+S_{12,2}=\text { L.H.S. of (ii). }
\end{aligned}
$$

By a similar argument it can be shown that (iii) is true. Therefore, $\left(M^{4}, g\right)$ is a (WCRS $)_{4}$ which is neither Ricci recurrent nor (WRS $)_{4}$. Hence we can state the following:

TheOREm 6.1. Let $\left(M^{4}, g\right)$ be a Riemannian manifold endowed with the metric given by

$$
d s^{2}=g_{i j} d x^{i} d x^{j}=e^{x^{1}}\left(d x^{1}\right)^{2}+\left(x^{1}\right)^{2}\left(d x^{2}\right)^{2}+\left(x^{1} \sin x^{2}\right)^{2}\left(d x^{3}\right)^{2}+p^{2}\left(d x^{4}\right)^{2}
$$

$(i, j=1,2,3,4)$, where $p$ is an arbitrary constant and $0<x^{1}<1$. Then $\left(M^{4}, g\right)$ is a (WCRS $)_{4}$ with non-vanishing scalar curvature which is neither Ricci recurrent nor (WRS) $)_{4}$.

EXAMPLE 2. We define a Riemannian metric $g$ on $\mathbb{R}^{4}$ by

$$
d s^{2}=g_{i j} d x^{i} d x^{j}=(1+2 q)\left[\left(d x^{1}\right)^{2}+\left(d x^{2}\right)^{2}+\left(d x^{3}\right)^{2}+\left(d x^{4}\right)^{2}\right]
$$

$(i, j=1,2,3,4)$, where $q=e^{x^{1}} / k^{2}$ and $k$ is a non-zero constant. Then the only non-vanishing components of the Christoffel symbols and the curvature tensor are

$$
\begin{aligned}
\Gamma_{22}^{1} & =\Gamma_{33}^{1}=-\frac{q}{1+2 q}, \quad \Gamma_{11}^{1}=\Gamma_{12}^{2}=\Gamma_{13}^{3}=\frac{q}{1+2 q}, \\
R_{1221} & =R_{1331}=R_{1441}=\frac{q}{1+2 q}
\end{aligned}
$$

and the components which can be obtained from these by symmetry properties. Using the above relations, we find that the non-vanishing components of the Ricci tensor and their covariant derivatives are as follows:

$$
\begin{aligned}
S_{11} & =\frac{3 q}{(1+2 q)^{2}}, & S_{22} & =\frac{q}{(1+2 q)^{2}}, \\
S_{33} & =\frac{q}{(1+2 q)^{2}}, & S_{44} & =\frac{q}{(1+2 q)^{2}}, \\
S_{11,1} & =\frac{3 q(1-4 q)}{(1+2 q)^{3}}, & S_{22,1} & =\frac{q(1-4 q)}{(1+2 q)^{3}}, \\
S_{33,1} & =\frac{q(1-4 q)}{(1+2 q)^{3}}, & S_{44,1} & =\frac{q(1-4 q)}{(1+2 q)^{3}} .
\end{aligned}
$$

Also it can be easily shown that the scalar curvature of the resulting manifold $\left(M^{4}, g\right)$ is $r=6 q /(1+2 q)^{3}$, which is non-vanishing and non-constant. We 
shall now show that $M^{4}$ is a (WCRS $)_{4}$. The associated 1-forms are as follows:

$$
\begin{aligned}
& A_{i}(x)= \begin{cases}\frac{1-4 q}{1+2 q} & \text { for } i=1, \\
0 & \text { otherwise, }\end{cases} \\
& B_{i}(x)= \begin{cases}\frac{2}{1+2 q} & \text { for } i=1, \\
0 & \text { otherwise, }\end{cases} \\
& C_{i}(x)= \begin{cases}-\frac{8 q}{1+2 q} & \text { for } i=1, \\
0 & \text { otherwise }\end{cases}
\end{aligned}
$$

at any point $x \in M$. Now, (1.2) reduces to the equations

$$
\begin{aligned}
& S_{11,1}+S_{11,1}+S_{11,1}=A_{1} S_{11}+B_{1} S_{11}+C_{1} S_{11}, \\
& S_{22,1}+S_{21,2}+S_{12,2}=A_{1} S_{22}+B_{2} S_{21}+C_{2} S_{12}, \\
& S_{33,1}+S_{31,3}+S_{13,3}=A_{1} S_{33}+B_{3} S_{31}+C_{3} S_{13}, \\
& S_{44,1}+S_{41,4}+S_{14,4}=A_{1} S_{44}+B_{4} S_{41}+C_{4} S_{14},
\end{aligned}
$$

since for the other cases (1.2) holds trivially. From (6.6)-(6.8) we get

$$
\begin{aligned}
\text { R.H.S. of (i) } & =A_{1} S_{11}+B_{1} S_{11}+C_{1} S_{11}=\frac{9 q(1-4 q)}{(1+2 q)^{3}} \\
& =S_{11,1}+S_{11,1}+S_{11,1}=\text { L.H.S. of (i). }
\end{aligned}
$$

By a similar argument it can be shown that (ii)-(iv) are true. Therefore, $\left(M^{4}, g\right)$ is a (WCRS $)_{4}$ which is neither Ricci recurrent nor (WRS $)_{4}$. Thus we can state the following:

Theorem 6.2. Let $\left(M^{4}, g\right)$ be a Riemannian manifold endowed with the metric given by

$$
d s^{2}=g_{i j} d x^{i} d x^{j}=(1+2 q)\left[\left(d x^{1}\right)^{2}+\left(d x^{2}\right)^{2}+\left(d x^{3}\right)^{2}+\left(d x^{4}\right)^{2}\right]
$$

$(i, j=1,2,3,4)$, where $q=e^{x^{1}} / k^{2}$ and $k$ is a constant. Then $\left(M^{4}, g\right)$ is a (WCRS) ${ }_{4}$ which is neither Ricci recurrent nor (WRS) $)_{4}$.

Example 3. We define a Riemannian metric $g$ on $\mathbb{R}^{4}$ by

$$
d s^{2}=g_{i j} d x^{i} d x^{j}=\left(x^{4}\right)^{\frac{4}{3}}\left[\left(d x^{1}\right)^{2}+\left(d x^{2}\right)^{2}+\left(d x^{3}\right)^{2}\right]+\left(d x^{4}\right)^{2}
$$

$(i, j=1,2,3,4)$. Then the only non-vanishing components of the Christoffel symbols and the curvature tensor are

$$
\begin{aligned}
\Gamma_{14}^{1} & =\Gamma_{24}^{2}=\Gamma_{34}^{3}=\frac{2}{3 x^{4}}, \quad \Gamma_{11}^{4}=\Gamma_{22}^{4}=\Gamma_{33}^{4}=-\frac{2}{3}\left(x^{4}\right)^{1 / 3}, \\
R_{1441} & =R_{2442}=R_{3443}=\frac{2}{9\left(x^{4}\right)^{2 / 3}}
\end{aligned}
$$


and the components obtained by symmetry properties. The non-vanishing components of the Ricci tensor and their covariant derivatives are:

$$
\begin{aligned}
S_{11} & =\frac{2}{9\left(x^{4}\right)^{2 / 3}}, & S_{22} & =\frac{2}{9\left(x^{4}\right)^{2 / 3}}, \\
S_{33} & =\frac{2}{9\left(x^{4}\right)^{2 / 3}}, & S_{44} & =\frac{2}{3\left(x^{4}\right)^{2}}, \\
S_{11,4} & =-\frac{4}{9\left(x^{4}\right)^{2 / 3}}, & S_{22,4} & =-\frac{4}{9\left(x^{4}\right)^{2 / 3}}, \\
S_{33,4} & =-\frac{4}{9\left(x^{4}\right)^{2 / 3}}, & S_{44,4} & =-\frac{4}{3\left(x^{4}\right)^{3}} .
\end{aligned}
$$

It can be easily shown that the scalar curvature of the resulting manifold $\left(M^{4}, g\right)$ is $r=4 / 3\left(x^{4}\right)^{2}$, which is non-vanishing and non-constant. We shall now show that $M^{4}$ is a (WCRS $)_{4}$. The associated 1 -forms are

$$
\begin{aligned}
& A_{i}(x)= \begin{cases}-\frac{2}{x^{4}} & \text { for } i=4, \\
0 & \text { otherwise, }\end{cases} \\
& B_{i}(x)= \begin{cases}-\frac{1}{x^{4}} & \text { for } i=4, \\
0 & \text { otherwise, }\end{cases} \\
& C_{i}(x)= \begin{cases}-\frac{3}{x^{4}} & \text { for } i=4, \\
0 & \text { otherwise }\end{cases}
\end{aligned}
$$

at any point $x \in M$. Now, (1.2) reduces to the equations

$$
\begin{aligned}
& S_{11,4}+S_{14,1}+S_{41,1}=A_{4} S_{11}+B_{1} S_{14}+C_{1} S_{41}, \\
& S_{22,4}+S_{24,2}+S_{42,2}=A_{4} S_{22}+B_{2} S_{24}+C_{2} S_{42}, \\
& S_{33,4}+S_{34,3}+S_{43,3}=A_{4} S_{33}+B_{3} S_{34}+C_{3} S_{43}, \\
& S_{44,4}+S_{44,4}+S_{44,4}=A_{4} S_{44}+B_{4} S_{44}+C_{4} S_{44},
\end{aligned}
$$

since for the other cases (1.2) holds trivially. By (6.10)-(6.12) we get

$$
\begin{aligned}
\text { R.H.S. of (i) } & =A_{4} S_{11}+B_{1} S_{14}+C_{1} S_{41}=-\frac{4}{9\left(x^{4}\right)^{5 / 3}} \\
& =S_{11,4}+S_{14,1}+S_{41,1}=\text { L.H.S. of (i). }
\end{aligned}
$$

By a similar argument it can be shown that (ii)-(iv) are true. Therefore, $\left(M^{4}, g\right)$ is a (WCRS $)_{4}$ which is neither Ricci recurrent nor (WRS $)_{4}$. Thus we can state the following:

TheOREM 6.3. Let $\left(M^{4}, g\right)$ be a Riemannian manifold endowed with the metric given by

$$
d s^{2}=g_{i j} d x^{i} d x^{j}=\left(x^{4}\right)^{4 / 3}\left[\left(d x^{1}\right)^{2}+\left(d x^{2}\right)^{2}+\left(d x^{3}\right)^{2}\right]+\left(d x^{4}\right)^{2}
$$


$(i, j=1,2,3,4)$. Then $\left(M^{4}, g\right)$ is a $(\mathrm{WCRS})_{4}$ which is neither Ricci recurrent nor $(\mathrm{WRS})_{4}$.

EXAMPLE 4 . Let $M$ be an open subset of $\mathbb{R}^{n}(n \geq 4)$ endowed with the metric

$$
d s^{2}=g_{i j} d x^{i} d x^{j}=\left[\left(x^{4}\right)^{4 / 3}-1\right]\left[\left(d x^{1}\right)^{2}+\left(d x^{2}\right)^{2}+\left(d x^{3}\right)^{2}\right]+\delta_{a b} d x^{a} d x^{b}
$$

$(i, j=1, \ldots, n)$, where $\delta_{a b}$ is the Kronecker delta and $a, b$ run from 1 to $n$.

Then the only non-vanishing components of the Christoffel symbols and the curvature tensor are

$$
\begin{aligned}
\Gamma_{14}^{1} & =\Gamma_{24}^{2}=\Gamma_{34}^{3}=\frac{2}{3 x^{4}}, \quad \Gamma_{11}^{4}=\Gamma_{22}^{4}=\Gamma_{33}^{4}=-\frac{2}{3}\left(x^{4}\right)^{1 / 3}, \\
R_{1441} & =R_{2442}=R_{3443}=\frac{2}{9\left(x^{4}\right)^{2 / 3}}
\end{aligned}
$$

and the components obtained by symmetry properties. Now the non-vanishing components of the Ricci tensor and their covariant derivatives are

$$
\begin{aligned}
S_{11} & =S_{22}=S_{33}=\frac{2}{9\left(x^{4}\right)^{2 / 3}}, \quad S_{44}=\frac{2}{3\left(x^{4}\right)^{2}}, \\
S_{11,4} & =-\frac{4}{9\left(x^{4}\right)^{2 / 3}}, \quad S_{22,4}=-\frac{4}{9\left(x^{4}\right)^{2 / 3}}, \\
S_{33,4} & =-\frac{4}{9\left(x^{4}\right)^{\frac{2}{3}}}, \quad S_{44,4}=-\frac{4}{3\left(x^{4}\right)^{3}},
\end{aligned}
$$

It can be easily shown that the scalar curvature of the resulting manifold $\left(M^{n}, g\right)$ is $r=4 / 3\left(x^{4}\right)^{2}$, which is non-vanishing and non-constant. We shall now show that this $M^{n}$ is a (WCRS $)_{n}$.

The associated 1-forms are

$$
\begin{aligned}
& A_{i}(x)= \begin{cases}-\frac{2}{x^{4}} & \text { for } i=4 \\
0 & \text { otherwise }\end{cases} \\
& B_{i}(x)= \begin{cases}-\frac{3}{x^{4}} & \text { for } i=4 \\
0 & \text { otherwise }\end{cases} \\
& C_{i}(x)= \begin{cases}-\frac{1}{x^{4}} & \text { for } i=4 \\
0 & \text { otherwise }\end{cases}
\end{aligned}
$$

at any point $x \in M$. Then proceeding as in Example 3, it can be easily shown that the manifold under consideration is a (WCRS) ${ }_{n}$ which is neither Ricci recurrent nor $(\mathrm{WRS})_{n}$. Hence we can state the following: 
Theorem 6.4. Let $\left(M^{n}, g\right)(n \geq 4)$ be a Riemannian manifold endowed with the metric given by

$$
d s^{2}=g_{i j} d x^{i} d x^{j}=\left[\left(x^{4}\right)^{4 / 3}-1\right]\left[\left(d x^{1}\right)^{2}+\left(d x^{2}\right)^{2}+\left(d x^{3}\right)^{2}\right]+\delta_{a b} d x^{a} d x^{b}
$$

$(i, j=1, \ldots, n)$, where $\delta_{a b}$ is the Kronecker delta and $a, b$ run from 1 to $n$. Then $\left(M^{n}, g\right)(n \geq 4)$ is a (WCRS $)_{n}$ which is neither Ricci recurrent nor $(\mathrm{WRS})_{n}$.

Acknowledgements. The authors express their sincere thanks to the referee for his valuable suggestions leading to the improvement of the paper.

\section{References}

[1] M. C. Chaki and S. Koley, On generalized pseudo Ricci symmetric manifolds, Period. Math. Hungar. 28 (1994), 123-129.

[2] M. Novello and M. J. Reboucas, The stability of a rotating universe, Astrophys. J. 225 (1978), 719-724.

[3] J. A. Schouten, Ricci Calculus, 2nd ed., Springer, Berlin, 1954.

[4] L. Tamássy and T. Q. Binh, On weak symmetries of Einstein and Sasakian manifolds, Tensor (N.S.) 53 (1993), 140-148.

[5] Y. Watanabe, Integral inequalities in compact orientable manifolds, Riemannian or Kählerian, Kōdai Math. Sem. Rep. 20 (1968), 264-271.

[6] K. Yano, Integral Formulas in Riemannian Geometry, Dekker, New York, 1970.

Department of Mathematics

University of Burdwan

Golapbag

Burdwan-713 104

West Bengal, India

E-mail: aask2003@yahoo.co.in

aask@epatra.com

Received 22.6.2006

and in final form 17.8.2006 